\title{
Transtorno obsessivo-compulsivo: possíveis subtipos
}

\author{
Ana Gabriela Hounie, Sergio de A Brotto, J uliana Diniz, Priscila J Chacon e \\ Eurípedes C M iguel
}

Departamento de Psiquiatria da Faculdade de Medicina da Universidade de São Paulo. São Paulo, SP, Brasil

\begin{abstract}
Several studies suggest that OCD is probably a heterogeneous disorder with several putative subtypes. Differences in treatment response, disease course, and clinical features are suggestive of the existence of subtypes with specific underlying pathophysiological substrates. Whether studying OCD from a phenomenological or genetic standpoint phenotypic characterization becomes crucial. This manuscript summarizes data that support the existence of a subgroup of $O C D$ related to tics, a group with an early age onset; a possible subgroup related to rheumatic fever; and a group with poor insight. Validation of these putative subtypes may be possible with genetic family studies and will contribute to the development of more specific treatments.
\end{abstract}

Keywords Obsessive-compulsive disorder. Phenotypes.

\section{Introdução}

Diversas pesquisas indicam que o transtorno obsessivo-compulsivo (TOC) é um transtorno heterogêneo. As diferentes taxas de resposta ao tratamento, os diferentes cursos evolutivos, as diversas formas de apresentação da síndrome sugerem a existência de diversos subtipos com possíveis bases fisiopatológicas específicas. No intuito de estudar o TOC, seja do ponto de vista fenomenológico ou genético, a caracterização de fenótipos clínicos mais precisos torna-se crucial. Neste artigo, enfatizam-se subgrupos delimitados de acordo com a presença de determinadas características consideradas importantes na expressão do fenótipo. Assim, salientar-se-ão as diferenças clínicas baseadas na presença ou não de tiques, na idade de início dos sintomas, na presença de febre reumática e na crítica do paciente (insight) em relação aos sintomas obsessivo-compulsivos.

\section{TOC associado a tiques}

Pacientes que, além do TOC, manifestam tiques crônicos ou a síndrome de Tourette (ST) parecem constituir um grupo separado com características específicas de resposta a tratamento, clínicas e fenomenológicas.

Esse grupo caracteriza-se por uma idade mais precoce de aparecimento dos sintomas, ${ }^{1}$ um perfil diferente de comorbidades, por afetar predominantemente o sexo masculino e apresentar um padrão distinto de resposta ao tratamento. ${ }^{2,3} \mathrm{Se}-$ gundo Coffey et al, ${ }^{4}$ pacientes com TOC e ST apresentaram taxas mais altas de comorbidades com transtorno de déficit de atenção com hiperatividade (TDAH), fobia social, tricotilomania e transtorno dismórfico corporal que as observadas nos pacientes com TOC, mas sem ST. Além disso, esse grupo apresenta maior frequiência de transtornos de humor e de ansiedade, comportamento disruptivo e abuso de substân- cias do que pacientes com ST ou TOC isoladamente, indicando maior gravidade (Tabela). ${ }^{4}$

Recentemente, estudos genéticos não só encontraram mais TOC clínico e subclínico em parentes de pacientes com ST como também freqüências mais altas de tiques ou ST em parentes de pacientes com TOC. ${ }^{5,6}$ Esses achados reforçam a idéia de que pelo menos algumas formas de TOC são etiologicamente relacionadas à $\mathrm{ST}^{6}$

Do ponto de vista fenomenológico, encontram-se diferenças entre os pacientes com TOC com e sem tiques quando avaliados com a Yale-Brown Obsessive Compulsive Scale (Y-BOCS). ${ }^{7}$ A Y-BOCS, escala mais utilizada para a caracterização dos sintomas obsessivo-compulsivos (SOC), constitui-se de oito categorias de obsessões (de agressão, contaminação, sexuais, colecionamento, religiosas, de simetria, somáticas e diversas) e de sete categorias de compulsões (limpeza, verificação, repetição, contagem, ordenação, colecionamento e diversas).

Embora a gravidade dos sintomas pareça não diferir entre os dois grupos, ${ }^{2}$ seu número e natureza podem ser discriminadores importantes. Por exemplo, preocupações de contaminação e compulsões de lavagem foram mais freqüentemente observadas em pacientes com TOC sem tiques, enquanto os pacientes com TOC e tiques são mais propensos a apresentar pensamentos ou imagens violentas e sexuais intrusivas, rituais de colecionamento e contagem. Compulsões "tic-like", ou seja, comportamentos semelhantes a tiques, porém precedidos de obsessões, foram mais freqüentemente encontradas em pacientes com TOC e tiques. ${ }^{1,8}$

Na tentativa de superar as limitações dos estudos que apenas categorizam a presença ou ausência de sintomas, foi proposta uma abordagem multidimensional para os sintomas obsessivos. Por meio de uma análise fatorial dos sintomas listados na 


\section{Tabela - Definições de fenômenos sensoriais.}

1. Sensações corporais: focais ou generalizadas, ocorrendo antes de o paciente realizar o comportamento repetitivo. Sensação tátil na pele: "eu uso um batom 20 vezes por dia para aliviar uma sensação de secura nos lábios". Sensações musculoesqueléticas ou viscerais: "quando eu como, sinto meu estômago aumentando, e isto me incomoda. Eu tenho uma sensação estranha nele e tenho que checar várias vezes como ele está para me livrar dessa sensação desconfortável".

2. Sensações mentais: sensações ou percepções gerais, desconfortáveis, que ocorrem antes ou durante a realização de um comportamento repetitivo. Necessidade apenas ou premência: "ter de" realizar um comportamento repetitivo; uma força ou impulso sem qualquer obsessão, medo, preocupação ou sensação corporal: "eu não sei o motivo que me leva a checar as portas. Eu não tenho nenhum pensamento ruim, imagem ou medo, eu apenas "tenho de voltar e checar várias e várias vezes".

Liberação de energia: uma sensação geral (não focal) de uma tensão interior ou pressão; uma energia mental que aumenta e precisa ser descarregada: "eu sinto uma tensão mental crescendo se eu não confiro os meus armários... Não é uma sensação física, sinto uma energia na minha mente... Eu tenho de checar para aliviar essa tensão".

Incompletude: uma sensação interna de incompletude, imperfeição ou insuficiência; uma sensação ou desconforto subjetivo: "pior que as obsessões é o sentimento de que sempre falta alguma coisa em mim. Muito raramente eu me livro desde sentimento quando eu faço algumas coisas várias e várias vezes". Percepções "just right": uma sensação geral ou percepção de não estar certo, como deve ser, ou "legal" e realizar um comportamento repetitivo até atingir essa sensação de estar OK, certo ou legal. Para muitos pacientes, essas sensações estão ligadas a modalidades sensoriais (como as coisas parecem visualmente ou como elas soam): "eu levo horas para me vestir por que as coisas têm de parecer visualmente certas ou quando eu toco minha roupa ela tem de ter uma textura certa. Eu tiro e coloco as roupas várias e várias vezes para que as coisas pareçam certas."

Y-BOCS, pesquisadores identificaram quatro dimensões de sintomas: (1) obsessões de agressão, sexuais, somáticas e religiosas e compulsões relacionadas; (2) obsessões e compulsões de simetria, ordenação e contagem; (3) obsessões de contaminação e compulsões de limpeza; e (4) obsessões e compulsões de colecionamento. Os fatores 1,2 e 4 foram mais freqüentemente encontrados em pacientes com TOC e tiques do que naqueles com TOC sem tiques. ${ }^{9}$

Também dentro da fenomenologia, outro modo de abordar a psicopatologia do TOC e da ST é a investigação das experiências subjetivas que acompanham os comportamentos repetitivos. Uma grande variedade dessas experiências foi descrita associada aos sintomas principais. Essas experiências são classificadas como cognitivas (pensamentos, idéias ou imagens), ansiedade autonômica (sensações somáticas de ansiedade) e fenômenos sensoriais (sensações subjetivas, sentimentos gerais, sensações corporais) (Tabela). ${ }^{10}$

Por meio de um instrumento específico (Entrevista de Comportamentos Repetitivos USP/Harvard) ${ }^{2}$ comparam-se as experiências subjetivas associadas aos sintomas dos pacientes com ST e TOC e TOC apenas. Estes relataram mais fenômenos cognitivos e de ansiedade autonômica e uma menor freqüência de fenômenos sensoriais precedendo seus comportamentos repetitivos. ${ }^{2}$

\section{Relação com a idade de início do TOC}

Estudos epidemiológicos revelam uma curva bimodal para a idade de início dos sintomas: os homens os apresentam numa idade mais precoce (na infância ou adolescência), enquanto as mulheres, mais na idade adulta. Casos de TOC com início precoce apresentam maior comorbidade com tiques, mais fenômenos sensoriais e mais compulsões "tic-like". $1{ }^{1 *}$ Além disso, segundo alguns estudos, ${ }^{12^{*}}$ esses pacientes apresentam pior prognóstico, com curso crônico e pior resposta ao tratamento com clomipramina, ao contrário do que ocorre com pacientes com início mais tardio. Dada a superposição do fenótipo desses dois grupos de pacientes, resta clarificar se são dois subgrupos distintos ou se, do ponto de vista genético e/ou epigenético, a mesma vulnerabilidade que predispõe os pacientes aos tiques os predispõe a desenvolver TOC precocemen- te. $\mathrm{O}$ mais recente estudo familiar de TOC encontrou um risco para TOC de $11,7 \%$ em familiares de primeiro grau. ${ }^{13}$ Muito interessante é o fato de que não foi encontrado nenhum caso de TOC entre os familiares de casos com TOC de início tardio (após os 18 anos), o que favorece o TOC de início precoce ser mais "familiar". Um achado ainda mais interessante ainda é o fato de que quanto maior o número de casos de TOC em uma mesma família, mais precoce foi o seu início no caso-índice.

\section{TOC associado à febre reumática}

A febre reumática (FR) é caracterizada por manifestações sistêmicas (cardite e artrite) e no sistema nervoso central (coréia de Sydenhamn - CS) desencadeadas por uma infecção estreptocócica orofaríngea. Dentre as manifestações psicopatológicas, pesquisas recentes destacam a ocorrência de sintomas obsessivo-compulsivos. ${ }^{14}$

Teorias sobre a fisiopatologia da CS envolvem anormalidades no processo imunológico e incluem agressão de células-T ou anticorpos antineuronais contra gânglios da base. ${ }^{15}$ Existem evidências de que os gânglios da base estejam envolvidos no TOC, nos tiques e na ST assim como no THDA. ${ }^{16}$ É possível que, em alguns casos, a expressão fenotípica possa refletir o local da agressão (imunológica ou não) dentro dos gânglios da base ou dos circuitos corticoestriatais. Dessa forma, lesões envolvendo o caudado poderiam levar a obsessões e compulsões, enquanto lesões no putâmen seriam seguidas de tiques. ${ }^{17}$

Fatores relacionados à susceptibilidade do paciente também podem ser relevantes. De interesse particular é a descrição de um anticorpo monoclonal que funciona como marcador para FR. Esse anticorpo reconhece uma proteína (D8/17) na superfície dos linfócitos $\mathrm{B}$ mais freqüientemente nos pacientes com $\mathrm{FR}$ do que na população normal. ${ }^{18}$ Embora ainda não seja claro o papel desse marcador na fisiopatologia de doenças pós-estreptocócicas, a alta percentagem do D8/17 em alguns pacientes com TOC ou ST ${ }^{19}$ tem levado a considerar a hipótese de que ele possa vir a servir como marcador genético de predisposição a esses transtornos.

Esses achados fazem sentido no contexto de recentes estudos que levantam a possibilidade de que sintomas obsessivocompulsivos, tiques e hiperatividade motora possam ser se-

*Rosario-Campos MC, Leckman JF, Mercadante MT, Shavitt RG, Prado HS, Sada P, et al. Early-onset obsessive-compulsive disorder as a possible subgroup. Am J Psychiatry (in press). 
qüelas da infecção estreptocócica e de suas conseqüentes anormalidades imunológicas, mesmo na ausência de sintomas clínicos da FR ou CS. Esses autores propuseram o acrônimo "Pandas" (Pediatric Autoimmune Neuropsychiatric Disorders Associated with Streptococcal Infection) para designar esse subgrupo de transtornos neuropsiquiátricos da infância. Esse subgrupo também apresenta maior expressão do D8/17 quando comparados a controles. Se a infecção estreptocócica e suas reações imunes anormais forem implicadas na determinação de subtipos clínicos do TOC, novos tratamentos poderão ser utilizados como, por exemplo, profilaxia com antibióticos, imunoterapia ou plasmaferese ${ }^{20}$ (ver artigo de Mercadante sobre aspectos neuroimunológicos neste volume).

\section{TOC com crítica prejudicada}

A capacidade crítica (insight) que o paciente apresenta em relação aos seus sintomas varia dentro de um continuum, desde uma crítica preservada, passando por idéias supervalorizadas a delirantes. ${ }^{21}$ Esses pacientes eram descritos anteriormente como portadores de estados esquizo-obsessivos ou psicoses obsessivas. ${ }^{23}$ Pacientes com prejuízo da crítica tendem a apresentar pior resposta a tratamento farmacológico e comportamental. ${ }^{21}$ Eisen et $a l,{ }^{23}$ entretanto, não encontraram correlação entre o grau de crítica e a resposta à sertralina em um estudo multicêntrico. Zohar et $\mathrm{al}^{24}$ associaram ao agente antiobsessivo a olanzapina, obtendo melhores resultados que a associação com neurolépticos comuns. Alguns autores propuseram que o TOC com crítica prejudicada (poor insight) seja considerado um subtipo diferente ou fazendo parte de um espectro esquizo-obsessivo. ${ }^{24}$

\section{Conclusão}

Diversos achados amparam a noção de que o TOC associado a tiques representa um dos possíveis subtipos clínicos, apresentando algumas características em comum com outros dois possí- veis subtipos: TOC de início precoce e TOC associado à FR. Todos eles têm um início precoce dos sintomas, são mais comuns em homens e mais freqüentemente associados a tiques. $\mathrm{O}$ padrão de sintomas também apresenta diversos fatores em comum, incluindo uma alta freqüência de fenômenos sensoriais. $\mathrm{O}$ grupo de autores do presente trabalho conduz atualmente um estudo em epidemiologia genética que objetiva verificar se existe agregação familiar de FR, TOC, tiques e THDA em familiares de primeiro grau de pacientes com FR. Espera-se verificar a existência de uma vulnerabilidade genética comum para TOC e FR, se a FR apenas contribui para modificar o fenótipo do TOC, ou se essa associação tem sido encontrada apenas por acaso, visto que são duas entidades mórbidas bastante prevalentes em países em desenvolvimento como o Brasil.

Como exposto acima, características clínicas podem ter implicações importantes para o tratamento. Por exemplo, em pacientes com TOC e tiques ou crítica prejudicada, a adição de neurolépticos ao esquema antiobsessivo está bem fundamentada. Pacientes com início precoce parecem ter pior prognóstico e merecem maior atenção em termos de políticas de saúde. Embora ainda limitado ao campo das pesquisas, é possível que um subtipo cujo desencadeamento e curso esteja associado à infecção estreptocócica possa responder a tratamentos imunológicos como a plasmaferese ou administração de imunoglobulinas. Para mais detalhes sobre os diferentes tratamentos dependentes das diversas características clínicas mencionadas no presente artigo, os artigos sobre tratamento encontrados nesta publicação podem ser consultados.

Este trabalho teve o apoio financeiro da Fundação de Amparo à Pesquisa do Estado de São Paulo (Processos no 98/15013-9 para Hounie AG e n ${ }^{\circ}$ 99/08560-6 para Miguel EC) e do Conselho Nacional de Desenvolvimento Científico e Tecnológico (Processo $\mathrm{n}^{\mathrm{o}}$ 521369/96-7 para Miguel EC).

\section{Referências}

1. Holzer JC, Goodman WK, McDougle CJ, Baer L, Boyarsky BK, Leckman JF, et al. Obsessive-compulsive disorder with and without a chronic tic disorder: A comparison of symptoms in 70 patients. Br J Psychiatry 1994;464:469-73.

2. Miguel EC, Baer L, Coffey BJ, Rauch SL, Savage CR, Sullivan RLO, et al. Phenomenological differences appearing with repetitive behaviours in obsessive-compulsive disorder and Gilles de la Tourette Syndrome. Brit J Psychiatry 1997; 170:140-5.

3. McDougle CJ, Goodman WK, Leckman JF, Lee NC, Heninger GR, Price LH. Haloperidol addition in fluvoxamine-refractory obsessivecompulsive disorder. A double blind, placebo-controlled study in patients with and without tics. Arch Gen Psychiatry 1994;51:303-8.

4. Coffey BJ, Miguel EC, Biederman J, Baer L, Rauch SL, Sullivan RLO, et al. Tourette s disorder with and without obsessive-compulsive disorder in adults: are they different? J Nerv Ment Dis 1998;186:201-6.

5. Pauls DL, Towbin KE, Leckman JF, Zahner JEP, Cohen DJ. Gilles de la Tourette s syndrome and obsessive-compulsive disorder evidence supporting a genetic relationship. Arch Gen Psychiatry 1986;43:1180-2.

6. Pauls DL, Alsobrook JP 2nd, Goodman W, Rasmussen S, Leckman JF. A family study of obsessive-compulsive disorder. Am J Psychiatry 1995;152:76-84.
7. Goodman WK, Price LH, Rasmussen SA, Mazure C, Fleischman RL, Hill CL, et al. The Yale-Brown obsessive-compulsive scale: I. development use and reliability. Arch Gen Psychiatry 1989;46:1006-11.

8. Leckman JF, Grice DE, Barr LC, Vries ALC, Martin C, Cohen DJ, et al. Tic-related vs non-tic-related obsessive-compulsive disorder. Anxiety 1995;1:208-15.

9. Leckman JF, Grice DE, Boardman J, Zhang H, Vitale A, Bondi C, et al. Symptoms of obsessive-compulsive disorder. Am J Psychiatry 1997; 154:911-7.

10. Miguel EC, Rosário-Campos MC, Prado HS, Valle RV, Rauch RL, Coffey, BJ, et al. Sensory Phenomena in Obsessive-Compulsive Disorder and Gilles de la Tourette syndrome. J Clin Psychiatry 2000;61:150-6.

11. Riddle MA, Scahill L, King R. Obsessive-compulsive disorder in children and adolescents: phenomenology and family history. J Am Acad Child Adolesc Psychiatry 1990;29:766-72.

12. Robins LN, Helzer JE, Weissman MM, Orvaschel H, Gruenberg E, Burke JD-Jr, et al: Lifetime prevalence of specific psychiatric disorders in three sites. Arch Gen Psychiatry 1984;41:959-67.

13. Nestadt G, Samuels J, Riddle M, Bienvenu J, Liang KY, LaBuda M et al. A family study of obsessive-compulsive disorder. Arch Gen Psychiatry 2000;57:358-63. 
14. Mercadante MT, Busatto GF, Lombroso PJ, Prado L, Rosario-Campos MC, Valle R, et al. The Psychiatric Symptoms of Rheumatic Fever. Am J Psychiatry 2000;157:2036-8.

15. Husby G. Antibodies reacting with citoplasm of subthalamic and caudate nuclei neurons in Chorea acute rheumatic fever. J Exp Med 1976;144:1094-110.

16. Castellanos FX, Giedd JN, Eckburg P, Marsh WL, Vaituzis AC, Kaysen $\mathrm{D}$, et al. Quantitative morphology of the caudate nucleus in attention deficit hyperactivity disorder. Am J Psychiatry 1994;151:1791-6.

17. Baxter LR, Schwartz JM, Bergman KS, Szuba MP, Guze BH, Mazziota JC, et al. audate glucose metabolic rate changes with both drug and behavior therapy for obsessive-compulsive disorder. Arch Gen Psychiatry 1992;49:681-.9.

18. Zabriskie JB, Lavenchy D, Williams RC Jr, Fu SM, Yeadon CA, Fotino $\mathrm{M}$, et al. Rheumatic fever associated B cell alloantigens as identified by monoclonal antibodies. Arthritis Rheum 1985;28:1047-51.

19. Murphy T, Goodman W, Fudge MW, Williams RC, Ayoub EM, Dalal M, et al. B Lymphocyte antigen D8/17: a peripheral marker for childhood-onset obsessive-compulsive disorder and Tourette syndrome? Am J Psychiatry 1997;154:402-7.
20. Swedo SE, Leonard HL, Mittleman BB, Allen AJ, Rapoport JL, Dow $\mathrm{SP}$, et al. Identification of children with pediatric autoimmune neuropsychiatric disorders associated with streptococcal infections by a marker associated with rheumatic fever. Am J Psychiatry 1997;154:110-2.

21. Neziroglu FA, Stevens KP, Yaryura Tobias JA. Overvalued Ideas and their impact on treatment outcome. Rev Bras Psiquiatria 1999;21:209-16.

22. Insel TR, Akiskal HS. Obsessive-compulsive disorder with psychotic features: a phenomenological analysis. Am J Psychiatry 1986;43:1527-33.

23. Eisen JL, Phillips KA, Baer L, Beer DA, Atala KD, Rasmussen AS. The Brown Assessment of Beliefs Scale (BABS): reliability and validity. Am J Psychiatry 1998;155:102-8.

24. Zohar J, Sasson Y, Amiaz R, Chopra M. Epidemiology and treatment in OCD and schizophrenia. Paper presented at the fourth international OCD Conference February 11-13, 2000 St Thomas US Virgin Islands.

Correspondência: Ana Gabriela Hounie

Instituto de Psiquiatria da FM/USP

Rua Dr. Ovídio Pires de Campos, s/nº - 05403-010 São Paulo, SP, Brasil

E-mail: anah@protoc.com.br 